\title{
OPTICALLY PUMPED LOW THRESHOLD ZnSe BASED LASERS WITH 2.8 ML CdSe ACTIVE REGION
}

\author{
S. Sorokin, A. Toropov, T. Shubina, A. Lebedev, I. Sedova, S. Ivanov \\ Ioffe Physico-Technical Institute of Russian Academy of Sciences \\ Politekhnicheskaya 26, St.-Petersburg 194021, Russia
}

AND A. WAAG

Universität Würzburg, Physikalisches Institut Am Hubland, 8700 Würzburg, Germany

Room-temperature optically pumped $(\mathrm{Zn}, \mathrm{Mg})(\mathrm{S}, \mathrm{Se}) /(\mathrm{Zn}, \mathrm{Cd}) \mathrm{Se}$ laser structures have been grown by molecular beam epitaxy. Using of alternatively-strained short-period superlattice waveguide results in low threshold power density values over the whole blue-green $(470-520 \mathrm{~nm})$ wavelength range. Incorporation of $\mathrm{CdSe}$ fractional monolayer active region provides more than fourfold further decrease in threshold with respect to quantum well laser structure. Optical and structural properties of laser structure with 2.8 monolayer CdSe are discussed in detail.

PACS numbers: 78.66.Hf, 78.45.th

\section{Introduction}

Despite the recent reduction of interest in II-VI blue lasers due to very fast progress in the development of long-lived III-nitride ones, the green range of a visible spectrum, corresponding to the highest human eye sensitivity, is hardly reached by the (In, Ga)N system due to the difficulties in obtaining pseudomorphic quantum well (QW) with high In content. Reduction of threshold current density as well as of extended defect density are the key points for an increase in II-VI green lasers lifetime. The using of ternary alternatively-strained (AS) $\mathrm{ZnSSe} / \mathrm{ZnCdSe}$ short-period superlattice (SL) waveguide grown by non-interrupted process allows one to solve both problems [1,2]. The AS SL is known to prevent active region from defect propagation. The SL waveguide also provides sufficient optical and improved electronic confinement at room temperature operation. Here we present a series of laser structures with the SL waveguides of different Cd content in QWs, varied within (0-0.27) range. The studies of single CdSe fractional monolayers (FMs) in $\mathrm{ZnSe}$ matrix have demonstrated a dramatic increase in luminescence intensity within the 2-3 monolayer (ML) CdSe nominal thickness range [3]. Therefore, we suppose that an introduction of the CdSe FM active region in the center 
of the SL waveguide can lead to a further decrease in a laser threshold. This paper presents studies of SL laser structure with $2.8 \mathrm{ML}$ CdSe FM active region, especially focusing on the laser characteristics. The employing of the CdSe FM active region provides additional freedom in structure design, allowing one to vary lasing wavelength independently of the SL well composition.

\section{Experiment}

The structures of optically pumped $(\mathrm{Zn}, \mathrm{Mg}, \mathrm{Cd})(\mathrm{S}, \mathrm{Se}) \mathrm{SCH}$ lasers studied here were grown by molecular beam epitaxy (MBE) on $\mathrm{GaAs}(100)$ substrates with a GaAs buffer layer. Compositional control of ternary and quaternary alloys and other growth conditions have been described elsewhere $[4,5]$. The substrate temperature was kept at $270^{\circ} \mathrm{C}$. Laser structures with CdSe FM active region consist of $20 \mathrm{~nm} \mathrm{ZnSe}$ buffer layer, a $0.5 \mu \mathrm{m}$ thick $\mathrm{ZnMgSSe}$ bottom cladding layer, a 28-period ( $3 \mathrm{~nm} \mathrm{ZnS} \mathrm{Zn}_{0.14} \mathrm{Se}_{0.86} / 5 \mathrm{~nm} \mathrm{ZnSe}$ ) SL waveguide surrounding a $10 \mathrm{~nm}$ wide ZnSe QW centered with a $2.8 \mathrm{ML}$ CdSe FM, and, finally, a $0.1 \mu \mathrm{m} \mathrm{ZnMgSSe}$ top cladding layer protected by a $5 \mathrm{~nm}$ ZnSe cap. Both conventional MBE and migration-enchanced epitaxy (MEE) techniques $[6,7]$ were used for the CdSe FM growth. The reference structures with the similar design contain the conventional 7-10 nm (Zn,Cd)Se QW with the same as in the SL well Cd content varied from 0.27 to 0 (pure $\mathrm{ZnSe}$ ). Sulphur content in the SLs is within the 0.13-0.16 range. The peculiarities of the SL design and growth have been reported in Ref. [1].

Different characterization techniques including photoluminescence (PL), PL excitation (PLE), double crystal X-ray diffraction (DXRD) and transmission electron microscopy (TEM) were used. The PL spectra were excited using $351 \mathrm{~nm}$ line from a cw $\mathrm{Ar}^{+}$laser. PLE measurements were performed using a tungsten lamp emission dispersed by a single-grating monochromator. Laser characteristics of optically pumped structures were studied using a nitrogen laser with a 8 ns pulse width. DXRD rocking curves were measured in a symmetric mode with a (004) reflection. An Si-crystal was used as a monochromator for a $\mathrm{Cu} K_{\alpha}$ radiation. The plan-view TEM characterization were performed using Philips EM420 electron microscope.

\section{Results and discussion}

All reference structures covering the wide spectral range demonstrated the room temperature threshold power density values of $\approx 55 \mathrm{~kW} / \mathrm{cm}^{2}$ at $\lambda \approx 470 \mathrm{~nm}$, $\approx 19.5 \mathrm{~kW} / \mathrm{cm}^{2}$ at $\lambda \approx 490 \mathrm{~nm}$, and $\approx 18 \mathrm{~kW} / \mathrm{cm}^{2}$ at $\lambda \approx 523 \mathrm{~nm}$. This values are among the lowest ever reported. The laser generation in these structures arises on the red side of PL peak.

A schematic diagram as well as double-crystal X-ray rocking curve of laser structure with the $2.8 \mathrm{ML}$ CdSe active region are presented in Fig. 1. A set of SL satellites is clearly seen. Their relatively high width can be explained by the $S$ gradient in the SL barrier layers and some variations of thickness of constituent layers along the growth axis. The average SL lattice parameter determined by zero-order SL peak position is close to that of GaAs substrate $(\approx 100 \operatorname{arcsec})$, which corresponds to a less than $1 \%$ error in sulphur content in a bulk $\mathrm{ZnSSe}$ layer. The SL period estimated from the $\Theta-2 \Theta$ rocking curve is $\approx 78 \AA$. In spite of 


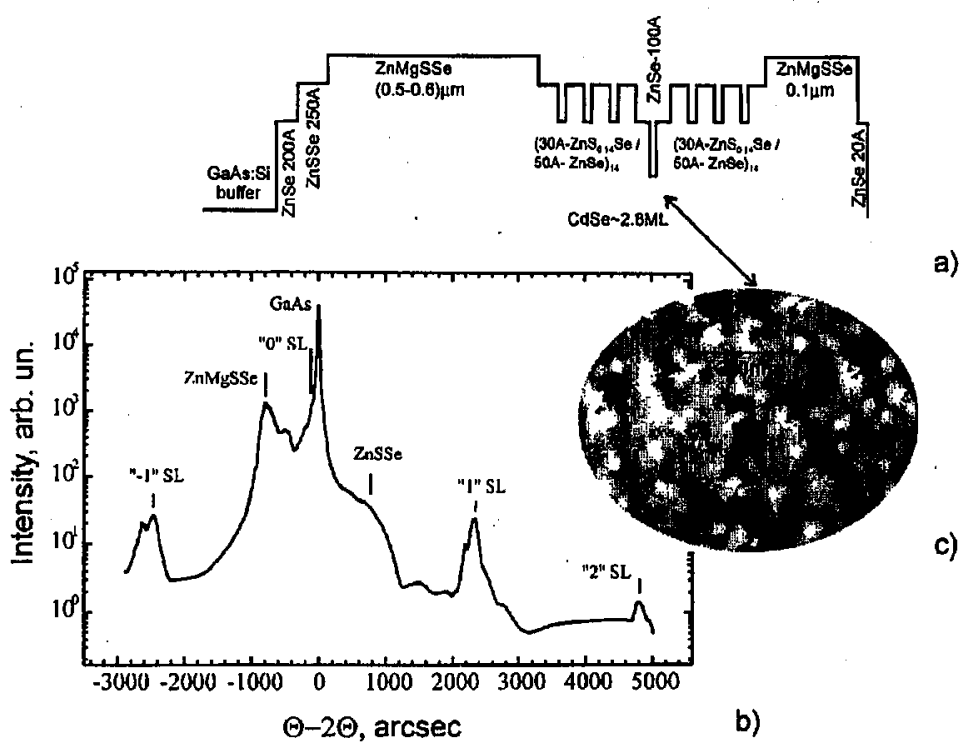

Fig. 1. Schematic diagram (a), double-crystal X-ray rocking curve (b) of laser structure with 2.8 ML CdSe FM active region and plan-view TEM image of CdSe FM (c).

noticeable lattice mismatch of $\mathrm{ZnMgSSe}$ claddings $\left(\Delta a / a \approx 6 \times 10^{-3}\right)$ the structure seems to be pseudomorphic that is confirmed by $\omega$-scanning XRD measurements. Note that the enhancement of the alternatively-strained structures stability to the compressive strain has been reported in [2]. Figure 1c shows the plan-view TEM image of $2.8 \mathrm{CdSe} F \mathrm{FM}$. This image has been obtained on a single $\mathrm{CdSe} / \mathrm{ZnSe}$ FM having the same CdSe nominal thickness as in the laser structure. A high density $\left(\approx 2 \times 10^{10} \mathrm{~cm}^{-2}\right)$ of dot-like objects with the average lateral size of $15-30 \mathrm{~nm}$ is clearly visible. Their density has been shown to increase with the CdSe nominal thickness [8]. We believe that these islands are not real electronic quantum dots, but rather small pieces of thin QWs with additional exciton localization potential which may result from $\mathrm{Cd}$ content and/or local thickness fluctuations. This interpretation agrees well with the large Stokes shift of the PL peak relatively to the PLE heavy-hole exciton peak in the FM structure (Fig. 2a). It is well known that Stokes shift in conventional QW structures is explained by a luminescence from low-energy states associated with local variation of $Q W$ width [9]. In the reference QW structure with the same PL maximum energy (Fig. 2b) the Stokes shift is of $\approx 10 \mathrm{meV}$.

Figure 2 presents the low-temperature PL and PLE spectra of the FM structure and of the reference QW structure. The CdSe FM PL band is much broader $(\approx 80 \mathrm{meV})$ and inhomogeneous, and most probably involves two peaks as have been reported in [6]. Contrary to the spectra of the QW structure, the PLE spectra of the FM structure depend on the registration energy marked by arrows in Fig. 2. Moreover, there is a well-pronounced peak, similar to the one observed in [10], especially when registered at the blue side of the FM PL contour, depending on 


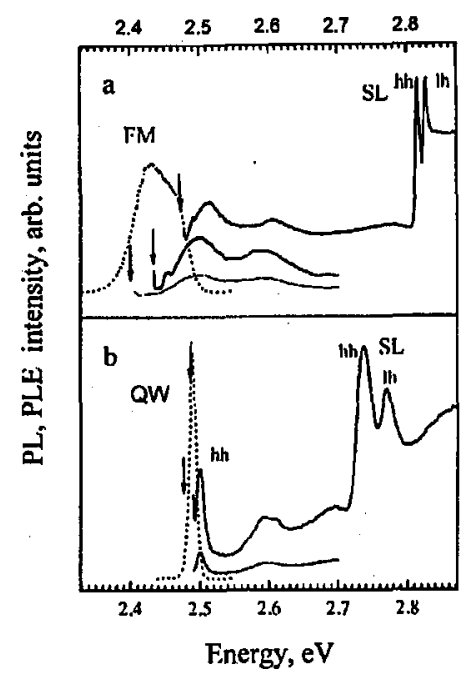

Fig. 2. Low-temperature PL (dotted lines) and PLE (solid lines) spectra of the FM structure (a) and of the reference QW structure (b).

the registration energy and separated from it by $\approx(22-26) \mathrm{meV}$. That may be correlated with the fine structure of the localized exciton spectrum which is known to arise from the anisotropy of localization potential in CdSe system [11].

The structure demonstrates a dramatic, more than fourfold decrease in the threshold power density $\left(3.9 \mathrm{~kW} / \mathrm{cm}^{2}\right.$ at $\left.\lambda \approx 510 \mathrm{~nm}, 300 \mathrm{~K}\right)$ with respect to the reference structure, emitting at the same wavelength $\left(\approx 18 \mathrm{~kW} / \mathrm{cm}^{2}\right)$. The spectra of the laser generation at room temperature as well as the temperature dependence of threshold power density for the FM laser structure are presented in Fig. 3. This dependence is obviously non-monotonous with the noticeable minimum at $\approx 100 \mathrm{~K}$, which may arise from both temperature changing of exciton localization mechanism and temperature enhanced vertical carrier transport through $\mathrm{ZnSe} / \mathrm{ZnSSe}$ waveguide SLs [12]. The role of the latter mechanism is confirmed by the disappearance of PL from SL at temperatures higher than $100 \mathrm{~K}$, whereas at low temperatures both CdSe FM and SL peaks are clearly seen. The FM structure exhibits only 2.5 -fold increase in the threshold from $5 \mathrm{~K}$ to $295 \mathrm{~K}$. The characteristic temperature at high temperatures is $\approx 175 \mathrm{~K}$. The laser demonstrates no noticeable degradation under the pumping power up to 25 -fold threshold during 24 hours. The laser generation in this structure arises on the blue side of PL peak in contrast to the reference ones.

The laser structure with $\approx 2$ ML CdSe FM insertion has been grown using MEE mode for the CdSe FM growth. This structure also demonstrates the low threshold power density (less than $10 \mathrm{~kW} / \mathrm{cm}^{2}$ ), but is characterized by a larger non-uniformity along wafer.

In summary, the introduction of 2-3 ML CdSe FM active region in the center of $\mathrm{ZnSe}$ QW surrounded by short period SL waveguide results in dramatic decrease in the threshold power density down to a $3.9 \mathrm{~kW} / \mathrm{cm}^{2}$ at room tempera- 


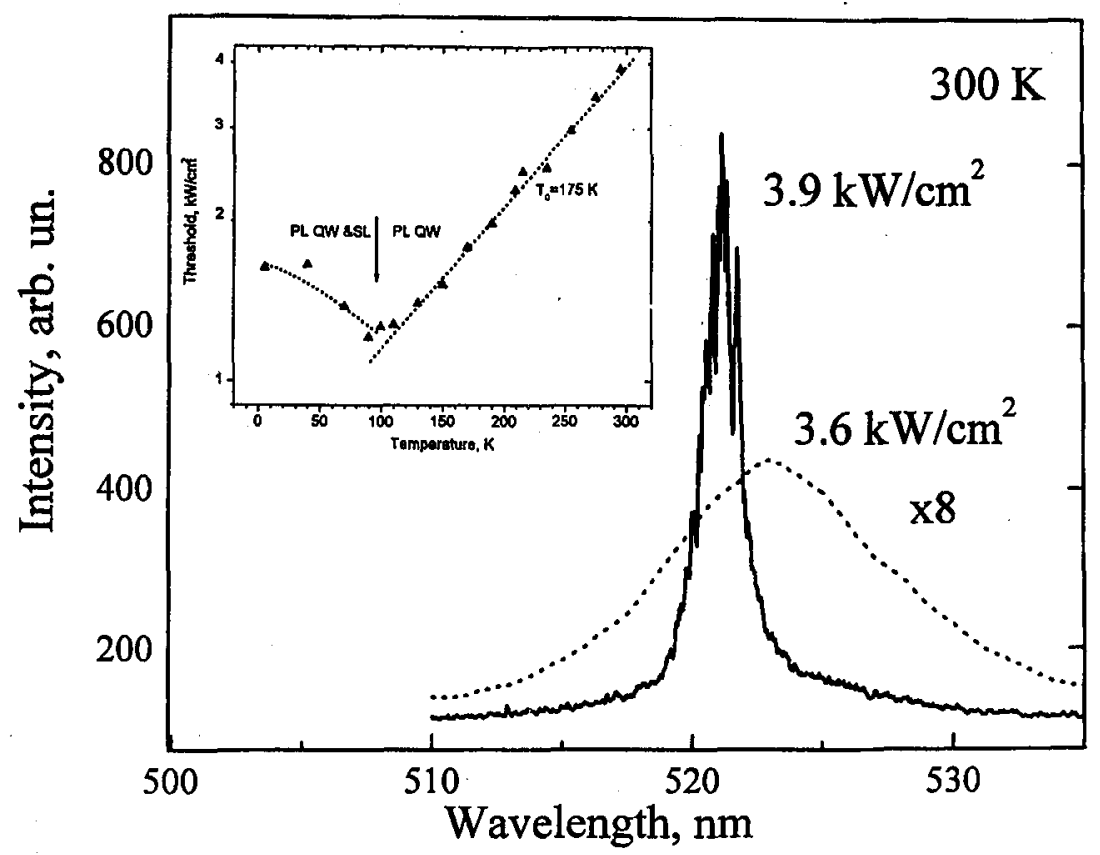

Fig. 3. The PL spectra below (dotted line) and above (solid line) threshold at room temperature together with temperature dependence of threshold power density (inset) for the FM laser structure.

ture, which is the lowest ever reported value for ZnSe-based lasers. The feasibility of technological realization, reasonable optical and carrier confinement, and a lack of noticeable degradation are very attractive for the injection device application.

\section{Acknowledgments}

The authors are thankful to A.A. Sitnikova and R.N. Kyutt for the TEM and XRD measurements, respectively. This work was supported in part by the Russian Foundation for Basic Research Grants and by the Program of Ministry of Science of RF "Physics of Solid-States Nanostructures".

\section{References}

[1] S. Sorokin, S. Ivanov, A. Toropov, T. Shubina, I. Sedova, M. Tkatchman, P. Kop'ev, Zh. Alferov, in: Proc. Int. Symp. "Nanostructures: Physics and Technology", St. Petersburg 1997, Ioffe Physico-Technical Institute, St. Petersburg 1997, p. 206.

[2] T.V. Shubina, S.V. Ivanov, A.A. Toropov, G.N. Aliev, M.G. Tkatchman, S.V. Sorokin, N.D. Il'inskaya, P.S. Kop'ev, J. Crystal Growth 184/185, 596 (1998).

[3] A.A. Toropov, S.V. Ivanov, T.V. Shubina, S.V. Sorokin, A.V. Lebedev, A.A. Sitnikova, P.S. Kop'ev, M. Willander, G. Pozina, J.P. Bergman, B. Monemar, to be published in Jpn. J. Appl. Phys., 1998. 
[4] S.V. Ivanov, S.V. Sorokin, P.S. Kop'ev, J.R. Kim, N.D. Jung, H.S. Park, J. Cryst. Growth 159, 16 (1996).

[5] S.V. Ivanov, S.V. Sorokin, I.L. Krestnikov, N.N. Faleev, B.Ya. Ber, I.V. Sedova, P.S. Kop'ev, J. Cryst. Growth 184/185, 70 (1998).

[6] S.V. Ivanov, A.A. Toropov, T.V. Shubina, S.V. Sorokin, A.V. Lebedev, I.V. Sedova, P.S. Kop'ev, G.R. Pozina, J.P. Bergman, B. Monemar, J. Appl. Phys. 83, 3168 (1998).

[7] S. Ivanov, A. Toropov, S. Sorokin, T. Shubina, I. Sedova, O. Lublinskaya, G. Pozina, B. Monemar, P.S. Kop'ev, in: Abstracts of the IIIrd Russian Conference on Physics of Semiconductors, Moscow 1997, Institute of Physics of RAS, Moscow 1997, p. 345.

[8] I. Sedova, T. Shubina, S. Sorokin, A. Sitnikova, A. Toropov, S. Ivanov, W. Willander, Abstracts of the XXVII International School on Physics of Semiconducting Compounds Jaszowiec '98, Ustron'-Jaszowiec (Poland) 1998.

[9] P.S. Kop'ev, I.N. Uraltsev, A.L. Efros, D.R. Yakovlev, A.V. Vinokurova, Semicond. 22, 424 (1988).

[10] F. Gindele, C. Markle, U. Woggon, W. Langbein, J.M. Hvam, K. Leonardi, K. Ohkawa, D. Hommel, J. Cryst. Growth 184/185, 306 (1998).

[11] M. Chamarro, C. Gourdon, Ph. Lavallard, O. Lublinskaya, A. Ekimov, Phys. Rev. $B$ 53, 1336 (1996).

[12] A. Lebedev, S. Sorokin, A. Toropov, T. Shubina, N. Il'inskaya, O. Nekrutkina, S. Ivanov, G. Pozina, P. Bergman, B. Monemar, Abstracts of the XXVII International School on Physics of Semiconducting Compounds Jaszowiec '98, Ustron'-Jaszowiec (Poland) 1998. 